Cita bibliográfica: García Alvarado, J. M., García Rodríguez, Mª P., y Pérez González, Ma E. (2018). Evaluación y medida del sellado de suelos en el Norte de Madrid (España). Boletín de la Asociación de Geógrafos Españoles, 76, 1-19. doi: 10.21138/bage.2513

\title{
Evaluación y medida del sellado de suelos en el Norte de Madrid (España)
}

Assessment and measurement of soil sealing in the north of Madrid (Spain)

\author{
José María García Alvarado
}

josemaga@ucm.es

Ma Pilar García Rodríguez

mpgarcia@ucm.es

\section{Mª Eugenia Pérez González}

meperez@ucm.es

Departamento de Análisis Geográfico Regional y Geografía Física

Universidad Complutense de Madrid (España)

\section{Resumen}

El sellado de suelos provocado por el crecimiento urbano produce una pérdida irreversible del ecosistema edáfico, que pocas veces es considerado en los proyectos de expansión urbanística. Aquí el proceso de consumo de suelo se analiza utilizando diversas fuentes de representación espacial, que son tratadas con técnicas de teledetección y SIG. Los nuevos proyectos urbanísticos del $\mathrm{N}$ de Madrid mantienen hasta la fecha entre el 41-64 \% del suelo sin sellar, minimizando los impactos negativos de la impermeabilización del suelo, si bien responde en parte a la ralentización de las obras durante la crisis. Este estudio establece una metodología de trabajo para detectar y medir la pérdida de suelos en cuatro PAUs en la Comunidad Autónoma de Madrid, como un ejemplo de trabajo a escala local. La accesibilidad de bases de datos, material cartográfico e imágenes de satélite facilita la actualización de la cartografía temática. Los PAUS han sido construidos en suelos que corresponden principalmente a Luvisoles, con inclusiones menores de Calcisoles, Leptosoles, Cambisoles y Regosoles. La baja capacidad agrológica de estos suelos, 
implica que el sellado de suelos no afecta a suelos muy fértiles, por lo que la pérdida agronómica no es muy importante.

Palabras clave: sellado de suelos; expansión urbana; teledección; SIG; cambio de escala.

\begin{abstract}
Soil sealing caused by urban growth leads to irreversible loss of soil ecosystem, which is rarely considered in urban development projects. Here the process of land consumption is analyzed using various sources of spatial representation that are treated with techniques of remote sensing and GIS. Nowadays new development projects in northern Madrid maintain a sealed soil about 41-64\%, reducing the negative impacts of land sealing, although it is partly a response to the slowdown in construction during the crisis. This study aims to establish a working methodology to detect and measure soil loss in four PAUs in the Madrid Autonomous Community, as a working example at a local scale. The accessibility of databases, cartographic material and satellite images facilitates thematic mapping updates. The PAUs have been built on soils mainly corresponding to Luvisols with minor inclusions of Calcisols, Leptosols, Cambisols and Regosol. The low agrological capacity of these soils means that the soil sealing does not affect very fertile soils and therefore there is no significant agronomic loss.
\end{abstract}

Key words: soil sealing; urban sprawl; remote sensing; GIS.

Agradecimientos: Este trabajo está financiado con el Proyecto del Ministerio de Economía y Competividad, CSO 2012-34785: "Sellado de Suelo en la Comunidad de Madrid y Áreas Limítrofes. Estudio Mediante Teledetección y Sistemas de Información Geográfica". IP: Ma Eugenia Pérez González.

\title{
1 Introducción
}

En 1992 el Ayuntamiento de Madrid comenzó a trabajar en la ampliación urbanística de la capital con la pretensión de lograr un diseño sostenible, que se materializó en diversos Programas de Actuación Urbanística (PAUS). Se perseguía generar áreas del entorno metropolitano, de alta calidad urbana y medioambiental, con bajas densidades y amplias zonas verdes que fomentasen la complejidad urbana a través de la integración de usos diversos y prevén la localización de diferentes equipamientos. Las más de 77000 viviendas previstas en la totalidad de desarrollos urbanísticos (Brandis, 2014) acogerían casi 235000 nuevos residentes. Los PAUS constituyeron una importante apuesta política que ha permitido desarrollar suelo urbanizado suficiente en las 
proximidades de la capital y crear nuevas zonas de expansión de la ciudad con la vocación de generar desarrollos equilibrados, equipados y autosuficientes.

La edificación de estos PAUS conlleva una pérdida de suelo por sellado, que debe ser analizada para medir sus posibles consecuencias medioambientales.

El concepto de sellado de suelos ha migrado desde sus inicios en los años setenta del pasado siglo, de ser un proceso físico más de degradación del suelo (FAO, 1979), hasta bien entrado el siglo XXI, en el que se incorpora la pérdida de suelo por impermeabilización antrópica. En el primer caso, el sellado del suelo sería un proceso intermedio entre la compactación (producida por pisoteo o paso de maquinaria sobre un suelo natural) y el encostramiento (Govers y Poesen, 1986; Poesen y Govers, 1986; Valentin, 1994), fase en la que el suelo pierde su actividad biológica y capacidad de infiltración. Estos suelos degradados por sellado serían todavía reversibles. Sin embargo, el sellado antrópico, producido por construcciones diversas, impermeabiliza el suelo y su pérdida es prácticamente irreversible. El equilibrio entre las necesidades e impactos derivados de crecimiento y mejora de la calidad de vida de la población se rompe cuando los proyectos urbanísticos responden únicamente a motivos económicos. Son numerosos los casos de consumo del suelo sobredimensionado ante presumibles demandas, que han dejado un paisaje fantasma, una vez rotado el ciclo económico de bonanza.

Ante la gravedad del problema la Comunidad Europea elaboró un documento de trabajo sobre directrices de limitación del sellado de suelo en el que establecería como medidas de atenuación de los efectos del sellado "la sustitución del cemento o el asfalto por materiales permeables, el apoyo de la «infraestructura verde», o un mayor recurso a los sistemas naturales de recogida del agua. Cuando las medidas de atenuación in situ resulten insuficientes, podría considerarse la adopción de medidas de compensación que mejoren las funciones del suelo en otros lugares" (European Commission, 2012).

Numerosos autores han escrito sobre la gravedad de este problema desde hace varias décadas (Eilers, 1973; Mualem y Assouline 1989; Heil et al., 1997; Netzband y Meinel, 1998; Singer y Bissonnais, 1998; Al Dousari et al., 2000; Burghardt, 2006; Pacual et al., 2005; García y Pérez 2007, 2011, 2014; Pulido et al., 2014), analizando la pérdida de suelo desde dimensiones espaciales muy diversas, desde análisis a nivel global o macroregional (Heil et al., 1997; Scalenghe y Marsan, 2009; Gardi et al., 2011), nacional (Munafo et al., 2010 y 2013) o regional y local (Xiao et al., 2013 y García et al., 2014). Algunos investigadores se adentran en las consecuencias medioambientales de este sellado, especialmente en relación a los movimientos del agua y al incremento de la erosión por aumento de la escorrentía (Mualem et al., 1990; Römkens et al., 1997; Ramos et al., 2000; Assouline y Mualem, 2006; Chen et al., 2013; Jakab et al., 2013; Ungaro et al., 2014) y a los cambios en la biomasa y la biodiversidad (Zhao et al., 2012). Otros 
autores plantean posibles estrategias para su protección (FAO, 1979; Prokop et al., 2011; Artmann, 2014).

En este estudio se quiere establecer una metodología de trabajo que ayude a la detección en cuatro áreas incluidas en el Programa de Actuación urbanística en la Comunidad de Madrid, y posterior medida del sellado de suelos, como un ejemplo de trabajo a escala local. La accesibilidad a bases de datos, material cartográfico e imágenes de satélite facilita la actualización de la cartografía temática. La evaluación de pérdida de suelo por crecimiento urbano se mide aquí a diferentes escalas, expresando los resultados por unidad de superficie total del PAU, lo que posibilita estudios evolutivos de este proceso, y a nivel parcelario, desglosando en cada una las diferentes proporciones de sellado.

El objetivo principal del trabajo es, por tanto, medir la pérdida de suelo por sellado en una unidad territorial concreta, a diferentes escalas, comparando distintas fuentes cartográficas. La elaboración de una adecuada base de datos permitirá en el futuro elaborar un índice de sellado, que establezca indicadores más precisos sobre la pérdida o consumo del suelo.

En este trabajo se intenta conocer la relación entre los desarrollos urbanos recientes y el sellado de suelo por afección urbana. La limitación, mitigación y/o compensación del sellado de suelo que señala la Agencia Medioambiental Europea (CE, 2012) en lo que al fenómeno complejo de dinámicas territoriales combinadas, factores y actores, generalmente de intereses contrapuestos, que constituye el crecimiento de la ciudad, no es tarea sencilla. La forma de ocupación, de transformación del suelo de sus usos y aprovechamientos, conceptual y metodológicamente, en relación con esas prácticas mitigadoras $-\mathrm{y}$ si fuese posible, y mejor, preventivas-, tiene por delante mucho trabajo metodológico y conceptual. Ahora solo se trata de una pequeña aportación al mismo, consistente en el análisis y comprensión del fenómeno particular de sellado, en unos casos concretos de crecimiento reciente, en la primera ciudad del país, esto es Madrid.

\section{2 Área de estudio}

Este trabajo se localiza al norte de la ciudad de Madrid y comprende cuatro planes de expansión urbanística, que de W a E reciben el nombre de Montecarmelo, Las Tablas, Sanchinarro y Valdebebas (Figura 1). Estos PAUS se han construido en un área de topografía muy suavemente ondulada, a una altitud alrededor de $660 \mathrm{~m}$ y constituida por materiales terciarios, fundamentalmente arcosas. El clima es mediterráneo, de marcada sequía estival, irregularidad pluviométrica, con torrencialidad acusada y matiz continental. La vegetación potencial predominante es perennifolia (Quercus ilex, Pinus sp., etc.) con estrato arbustivo xerófilo (Cistus ladanifer, Lavandula pedunculata, Rosmarinus officinalis, Thymus vulgaris, etc.). Esta vegetación ha sido en gran parte sustituida por cultivos de secano que, en la actualidad, ya han sido abandonados. Cabe destacar que las nuevas actuaciones urbanísticas en las que se contemplan amplios espacios 
abiertos, han diseñado parques urbanos utilizando especies arbóreo-arbustivas propias de los ecosistemas mediterráneos, con técnicas de regadío con bajo consumo hídrico y, por tanto, favoreciendo la preservación de las principales funciones del suelo.

Los suelos sobre los que se han edificado los PAUS de Montecarmelo, Sanchinarro y Las Tablas corresponden la mayoría a Luvisoles (asociación Luvisol cálcico y háplico) con pequeños enclaves de Calcicoles háplicos y Leptosoles eútricos. En Valdebebas además de la asociación antes señalada, también aparece la asociación de Cambisol eútrico, Luvisol háplico y Regosol dístrico.

\section{Figura 1. Área de estudio. Localización de los PAU en el municipio de Madrid}



Fuente: elaboración propia a partir del mapa base del Ayuntamiento de Madrid

Las capacidades agrológicas de estos suelos son bajas (Monturiol y Alcalá, 1990), por lo que el sellado no afecta a suelos muy fértiles y, en consecuencia la pérdida agronómica no es considerable, a diferencia de otros casos como los contemplados en la Bahía de Hangzhou (Xiao et al., 2013). La mayoría corresponden a la clase agrológica Ce2, en la que su uso está limitado por erosión e hidromorfía, aunque al situarse sobre pendientes menores al $8 \%$, el riesgo de erosión es muy ligero o moderado. Sólo en el área situada en el norte del PAU de Montecarmelo aparecen suelos más fértiles de clase Be1. Aun cuando el sellado de estos suelos no implica la pérdida de tierras muy fértiles, sin embargo pueden crear un importante problema ambiental al condicionar los movimientos del agua y modificar los ciclos biogeoquímicos. 


\section{Material y métodos}

Para la medida y evaluación de la pérdida de suelo por sellado se ha recopilado una amplia base de información espacial (imágenes de satélite, fotografías aéreas, cartografía temática digital y planos del catastro), que permite analizar este proceso a diferentes escalas. Los datos espaciales son tratados básicamente con dos softwares específicos: de teledetección (Erdas Imagine 2015), y de sistemas de información geográfica (ArcGis 2010). Asimismo se han analizado las propiedades físicas y químicas de algunas muestras superficiales de suelos para conocer qué tipo de materiales se están sellando.

Al iniciar el trabajo se seleccionaron las imágenes de satélite disponibles a través del Plan Nacional de Teledetección (www.ign.es) y de diversos servidores de acceso libre (http://glcf.umd.edu y http://glovis.usgs.gov/). Principalmente se analizaron imágenes de los satélites Spot-5 (de 8 de julio de 2013) y Landsat 5 y 8 (de 18 de agosto de 1984 y 16 de junio de 2013), pero estas últimas se han descartado con posterioridad porque la resolución espacial no era la más adecuada para medir el sellado a escala local, si bien resultan útiles para ver la evolución del suelo sellado en las últimas décadas (García y Pérez, 2016) y para la escala regional.

Para realizar una clasificación en la que medir el sellado de suelo se ha utilizado una imagen del satélite Spot-5, correspondiente a la escena 264-32, en el modo pancromático $(0,48-0,71 \mu \mathrm{m})$, con 2,5 m de resolución espacial, y multiespectral, con 10 metros para las bandas verde (0,50 $0,59 \mu \mathrm{m})$, rojo $(0,61-0,68 \mu \mathrm{m})$ e infrarrojo cercano $(0,78-0,89 \mu \mathrm{m})$ y $20 \mathrm{~m}$ para el infrarrojo medio $(1,58-1,75 \mu \mathrm{m})$.

En cuanto a los trabajos de SIG, la fuente empleada ha sido el mosaico de ortofotos de Plan Nacional de Ortofotografía Aérea, de las hojas 534-559, y el plano parcelario obtenido del servidor Cartociudad (Instituto Geográfico Nacional).

El tratamiento ha consistido en el establecimiento de cinco clases de niveles de sellado (de 0 a 4), y en la interpretación visual del nivel de sellado en cada una de las manzanas, con algunas comprobaciones de campo. De momento solo se ha establecido el nivel de sellado, las superficies de la manzanas y sus perímetros, con la intención de generar una base de datos, en las siguientes fases de la investigación, con más atributos, tanto físicos como ambientales en general.

Metodológicamente el trabajo tiene varias fases encaminadas al cálculo de la superficie sellada actual a diferentes escalas en varias manzanas urbanísticas del norte de Madrid. Esta medida se obtiene a partir de la clasificación supervisada de la imagen del satélite Spot y de la cartografía digital. Posteriormente se contrasta la calidad y fiabilidad de los diferentes cálculos y, por último, se integran los análisis del suelo en la cartografía obtenida para conocer cómo actúan las diferentes tipologías urbanas en la pérdida de suelos y/o en la reducción de las funciones bioedáficas. 
Los pasos seguidos con la imagen de satélite hasta obtener la superficie sellada son:

- Cortar la imagen a la superficie de los cuatro PAUS.

- Mejorar la resolución espacial de la imagen multiespectral (canales visibles e infrarrojos), mediante la mezcla con la banda pancromática.

- Seleccionar la mejor combinación de bandas para identificar visualmente los usos del suelo: edificaciones, infraestructuras, eriales, vegetación arbustiva y arbórea y suelos degradados o desnudos.

- Realizar clasificaciones supervisadas utilizando el algoritmo no paramétrico de máxima probabilidad.

- Evaluar las clasificaciones mediante la matriz de confusión

- Reclasificar errores y evaluación definitiva de las clasificaciones

- Medida de las diferentes superficies selladas de los PAUS y de los suelos sin sellar.

\section{Resultados y discusión}

El análisis comienza por una valoración, todo lo precisa que aconseje la escala de trabajo, cuantificable del sellado que ofrece la forma urbana y urbanística (usos y morfología y tramado o estructura) y cómo se han materializado estos nuevos desarrollos.

En este caso, Madrid, se optó como ensayo de la metodología y casos paradigmáticos del actual proceso de crecimiento urbano de la ciudad central, los cuatro Programas de Actuación Urbanística, en que se materializa el mayor crecimiento urbano de la ciudad en su expansión septentrional. Así, de oeste a este surgirán nuevos "espacios normativos" para usos fundamentalmente residenciales, combinados con algunos otros significativos o emblemáticos como la nueva Ciudad Deportiva del club de fútbol Real Madrid o de la frustrada Ciudad de la Justicia.

Estos PAUS se ubican en unos espacios intersticiales vacantes, segmentados por autopistas urbanas unas radiales y otras orbitales y por otros espacios de servidumbre (barreras ferroviarias), junto a otras piezas urbanas consolidadas en épocas anteriores, que constituyen antiguas barriadas. Estos casos son: Montecarmelo, Las Tablas, Sanchinarro y Valdebebas. Se han excluido los de Arroyo del Fresno, al oeste, y el del Ensanche de Barajas al este, por su menor significación y peso superficial. Desde que se aprobaran estos programas a finales del siglo XX, y al amparo del Plan General de Ordenación Urbana de Madrid de 1997, constituyen un significativo ejemplo de la forma de crecimiento de la ciudad central.

Es cierto que los PAUS del SE de Madrid, son de mayor envergadura, tanto por el número previsto de dotación de viviendas, como por las dimensiones superficiales. No obstante, el SE presenta unas peculiaridades que le hacen merecedor de un tratamiento aparte. 
Por la cronología de ocupación de los casos de estudio, hay que tener en cuenta los procesos acaecidos en el devenir económico y urbano de la ciudad y del país. Se inicia en pleno ciclo expansivo, conocido como "boom inmobiliario", incrementando la crónica demanda insatisfecha de vivienda en Madrid, elevándose los precios a cotas desconocidas en toda nuestra historia del mercado inmobiliario, dando lugar a una amenazante alza de todo lo relacionado con el sector de la edificación, que fue llamado "burbuja inmobiliaria". La crisis financiera y económica, en general, vino a acabar con esa situación, de forma que a partir de 2008, las expectativas de continuidad de la actividad de construcción en los PAUS quedaban en suspenso, manteniéndose ese parón, en la primera mitad de la segunda década del siglo XXI, años de referencia del mosaico de ortofoto utilizado y de las imágenes de satélite.

La elección de estos PAUS, pues el estancamiento de licitaciones de obra fue similar en todos ellos, se basa en la oportunidad que nos ofrece disponer de cuatro espacios urbanos, vivos en su dinámica transformadora de suelo y a una escala apropiada, ni lo suficientemente pequeña como para diluirnos en valores globales, ni tan grande como para perder la perspectiva cuando se trata de escala de proyecto u obra. Nos hemos movido a unas escalas aproximadas entre 1:50 000_ 1:20 000 en la presentación de Pau completo, y 1:10 000 como escalas de análisis y trabajo, con los problemas de resolución y dificultades de interpretación visual, que han tenido la fuente, y después se detallan. Efectivamente, más allá de 1:5000 se pierde la resolución y en la mayoría de los casos no se identifica ni forma ni uso.

La primera aproximación de la medida del sellado se realiza mediante la imagen del satélite Spot-5, pues su utilización permite el seguimiento y la casi continua actualización de la información obtenida. A partir de la clasificación supervisada de esta imagen se distinguen cuatro categorías de ocupación del suelo y se calcula la superficie sellada, que ha perdido todas sus propiedades edáficas, y la no sellada, que todavía mantiene las funciones bioedáficas. Las clases que se representan en la figura 2 son:

- Sellado 1: es el sellado más compacto (>80 \%, Valera et al., 2011) e incluye edificaciones en bloque, infraestructuras viarias de gran tamaño, centros comerciales, etc.

- Sellado 2: es un sellado con menor densidad (<80\%, Valera et al., 2011) y corresponde a viviendas unifamiliares, infraestructuras viarias de pequeño tamaño, campos de deportes, etc., en los que se mantienen parcialmente las funciones del suelo.

- No sellado 1: espacios ajardinados, parques, eriales, etc., aunque transformados conservan las funciones físico-químicas y biológicas del suelo.

- No sellado 2: suelos removidos y/o totalmente desnudos, que muestran gran compactación, pero todavía reversibles en sus funciones edáficas. 
Esta clasificación muestra el desarrollo integrado entre áreas edificadas y espacios verdes con desigual ocupación entre los diferentes PAUS. Para comprobar la fiabilidad de esta clasificación se ha realizado la matriz de confusión tomando 50 puntos de control en las cuatro categorías clasificadas. La fiabilidad es del 93,33\% (0,915 del coeficiente Kappa) mostrando algunos errores en el sellado 2 correspondiente a las carreteras, pues la imagen raster clasifica peor los elementos lineales. En las demás categorías el error es inferior al 1\%. No obstante siempre estará limitado por la escala de estudio, en torno a 1:50 000.

Figura 2. Clasificación supervisada de la imagen Spot 5, 8 de julio de 2013

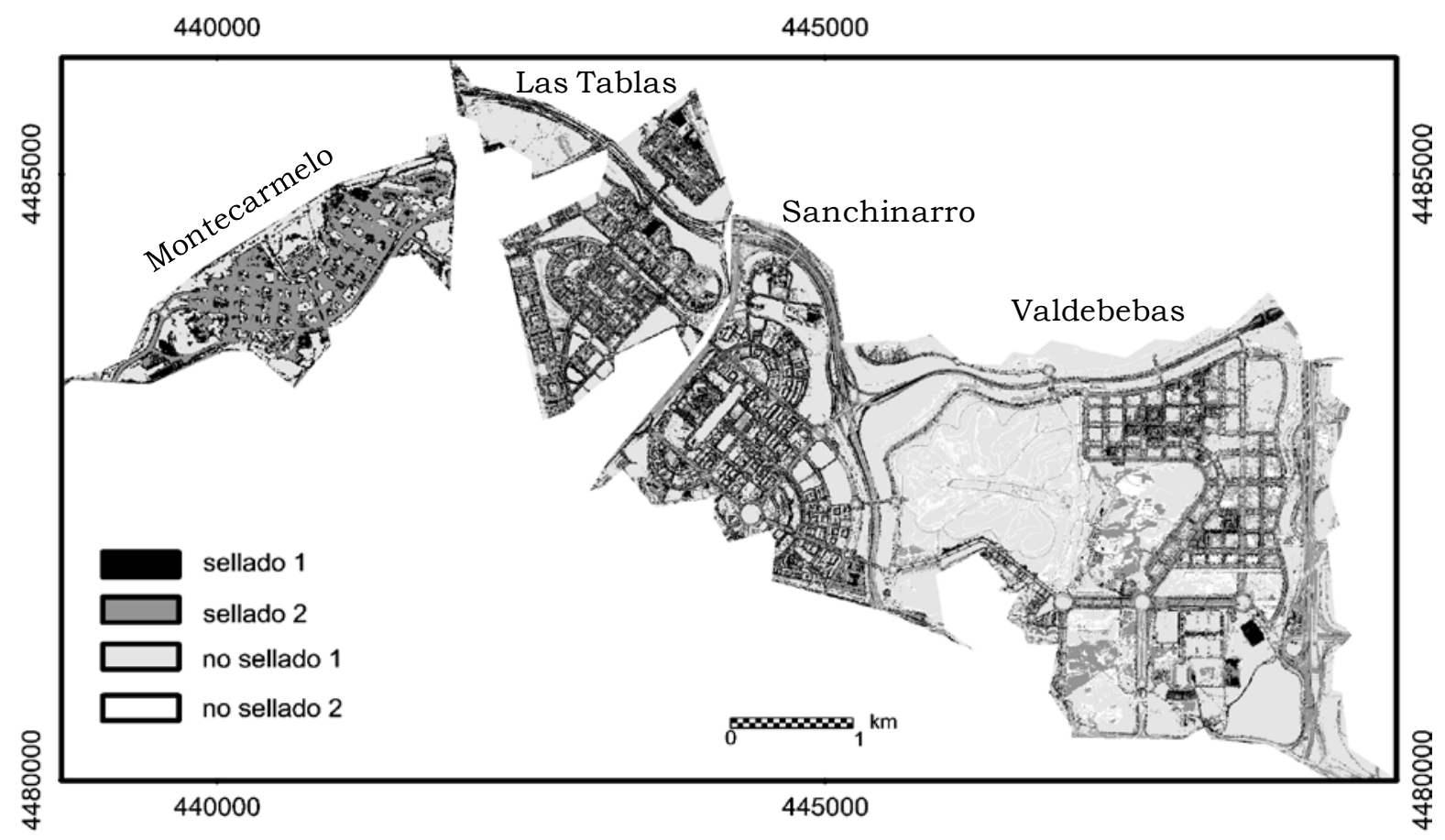

Fuente: elaboración propia a partir de la imagen Spot

En la Tabla 1 se resumen los valores obtenidos sobre la superficie sellada y no sellada en los cuatro PAUS, tanto en hectáreas como en porcentaje a partir de las clasificaciones supervisadas de las imágenes Spot. Tres de ellos tienen una superficie sellada que oscila entre el 45,6\% (Las Tablas) y el 59,3\% (Montecarmelo), mientras que el más reciente, Valdebebas, el sellado se reduce al $36,2 \%$, que no responde solo al retraso urbanístico durante la crisis, sino también al diseño de amplios espacios abiertos y extensos parques, pues alberga un amplio terreno, con 470 ha, que supondrá al finalizarse el mayor parque urbano de la ciudad de Madrid. 
Tabla 1. Superficie sellada y no sellada a partir de la imagen Spot

\begin{tabular}{|l|l|l|l|l|}
\hline & Montecarmelo & Las Tablas & Sanchinarro & Valdebebas \\
\hline Sellado (ha) & 157,0 & 157,5 & 224,9 & 400,8 \\
Sellado (\%) & 59,3 & 45,6 & 53,9 & 36,2 \\
\hline No sellado (ha) & 107,9 & 187,6 & 192,2 & 706,0 \\
No sellado (\%) & 40,7 & 54,4 & 46,1 & 63,8 \\
\hline
\end{tabular}

Fuente: elaboración propia

En una segunda fase se comparan estos datos con los obtenidos mediante SIG, lo que permite descender a una escala con mayor detalle (del 1:20 000 al 1:5000). En esta fase, al mosaico de ortofotos del Plan Nacional de Ortofotografía Aérea, de las hojas 534-559 se superpone a la parcelación de Cartociudad (IGN), si bien en algunos casos hubo de redibujar algunas manzanas, bien para ajustarlas entre ambas fuentes, bien para subdividir algunas manzanas que, siendo de grandes dimensiones una parte estaba totalmente sellada, y otra totalmente libre, lo que daría una media, correcta pero poco real.

A partir de ahí se clasificaron cada una de las 570 manzanas resultantes según su nivel de sellado. Las clases establecidas van desde menos del $20 \%$, a más de $80 \%$ de la superficie de la manzana sellada (Figura 3).

Sin que el porcentaje superficial de sellado por manzana sea el único criterio, sí es el básico para calificar una célula o trama urbana de cara al tema que nos ocupa. Hasta un $20 \%$ de suelo sellado en manzana urbana terminada, podría asumirse como una forma respetuosa de hacer ciudad, pues muestra un pequeño mal menor. El segundo intervalo de 20 a 40, todavía puede considerarse tolerable, entre 60 y 80, debería considerarse grave, y más del 80 \% debería ser clasificado como inasumible, y censurable, como mala práctica urbana. Sin duda, todo corte de intervalos tiene sus problemas, pero puede ser una correcta propuesta, para ayudar a valorar el cumplimiento de las directrices específicas de sellado de suelo y de cara a la aportación de este problema a la huella ecológica.

En definitiva, y de cara a desarrollos futuros, podrían contemplarse estos dos extremos, hasta $60 \%$ como límite admisible -aunque habría que conjugar también el sellado con los volúmenes de edificación-, y menos del 20 \% como deseable. No se nos oculta las repercusiones en el mercado del suelo, en el sector inmobiliario, etc., que esto supondría, así como en la "licuación" de la obra de fábrica urbana, y del plano final de los barrios, pero eso es otra vía de estudio.

De esta forma, el primer intervalo sería el ideal en el balance ecología/economía-mercado, el segundo óptimo, el tercero asumible con pérdida ecológica, y el cuarto inaceptable. Técnicos urbanistas, poderes públicos y opinión pública social, deberían consensuar en qué partes se cede, para ganar siempre en el largo plazo. 
A la vista de los datos anteriores podría parecer que el crecimiento de Madrid por el norte, ha optado por la primera de las opciones, y por lo tanto, por una forma de ciudad más sostenible, con pérdidas asumibles y con un elevado mantenimiento de suelo sin sellar. Sin que esto deje de ser cierto, no lo es en términos absolutos. El elevado valor de suelo sin sellar que mantiene las manzanas urbanas de esta parte de la ciudad, está sobredimensionado por un factor coyuntural, como es el parón constructivo, y el hecho de encontrarse un gran sector de estos PAUS sin terminar. 2012-2013 es un momento central de la crisis global y multicausal, con consecuencias en los tiempos y formas de las dinámicas urbanas. Muchas promociones previstas no llegaron a iniciarse, otras quedaron suspendidas, incluso suelos promocionados como usos terciarios, tales como los ocupados por la Ciudad de la Justicia. Hacia 2015, parece que se recupera el dinamismo, pero con una sustantiva bajada de precios.

\section{Tabla 2. Superficie de intervalos de sellado a partir de la ortofoto y valor promedio del tamaño de las manzanas urbanas}

\begin{tabular}{|c|c|c|c|c|c|c|c|c|c|}
\hline \multicolumn{2}{|c|}{ MONTECARMELO } & \multicolumn{2}{c|}{ LAS TABLAS } & \multicolumn{2}{c|}{ SANCHINARRO } & \multicolumn{2}{c|}{ VALDEBEBAS } \\
\hline \multicolumn{2}{|c|}{ Clase } & Superficie ha & $\%$ & Superficie ha & $\%$ & Superficie ha & $\%$ & Superficie ha & $\%$ \\
\hline 0 & $<20 \%$ & 93,45 & 47,8 & 112,93 & 53,9 & 127,97 & 55,9 & 709,9 & 72 \\
\hline 1 & $20-40 \%$ & 20,65 & 10,6 & 20,4 & 9,7 & 12,95 & 5,7 & 201,29 & 21 \\
\hline 2 & $40-60 \%$ & 8,81 & 4,5 & 0,86 & 0,4 & 19,49 & 8,5 & 41,71 & 4,2 \\
\hline 3 & $60-80 \%$ & 29,41 & 15,0 & 35,96 & 17,2 & 26,59 & 11,6 & 21,15 & 2,1 \\
\hline 4 & $>80$ & 43,31 & 22,1 & 39,41 & 18,8 & 41,86 & 18,3 & 9,8 & 1 \\
\hline \multicolumn{2}{|c|}{ Todas } & 195,63 & 100 & 209,56 & 100 & 228,87 & 100 & 983,85 & 100 \\
\hline \multicolumn{2}{|c|}{ Promedio } & 1,84 & & 1,49 & & 2,24 & & 4,35 & \\
\hline
\end{tabular}

Fuente: elaboración propia

En esta primera aproximación, una vez establecido los niveles de sellado, se ha generado una base de datos en que se contemplan a nivel de manzana, los atributos más básicos, como dimensión y perímetro, pero en sucesivos trabajos se irán incorporando otros aspectos tanto naturales, como de usos de suelo y morfología urbana.

En esta primera instancia sí, podemos indicar unos resultados preliminares:

1. Sin duda, comparando con zonas más centrales de la ciudad (García y Pérez, 2016), muestra una forma más abierta de trama urbana, lo que la hace compatible con un efecto de sellado menos severo. Es el caso de grandes superficies vinculadas a protección de infraestructuras viarias, algunos bulevares, glorietas y rotondas ajardinadas (Figura 4). Queda en evidencia la paradoja de que los PAUS con mayores instalaciones no residenciales generan en su entorno (y se refleja en su estadística), mayor porcentaje de suelo excluido de la amenaza de la impermeabilización del suelo. 
Figura 3. Sellado del suelo en los PAU del Norte de Madrid
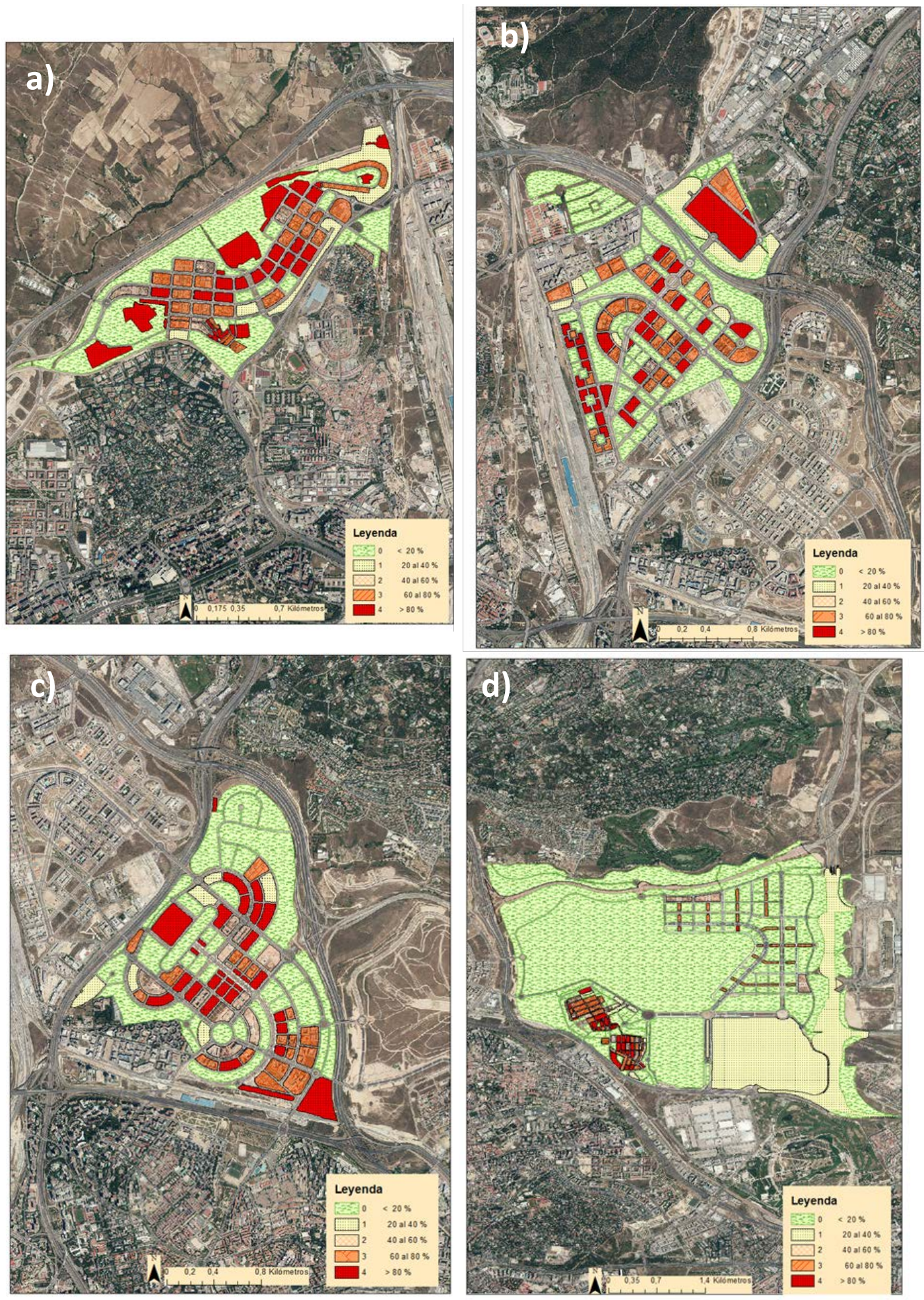

Leyenda: a) Montecarmelo, b) Las Tablas, c) Sanchinarro y d) Valdebebas

Fuente: elaboración propia a partir de la interpretación visual del PNOA 
Figura 4. Detalle de la cartografía del sellado a escala 1:5000 en el PAU de Las Tablas



Fuente: elaboración propia a partir del IGN

2. Valdebebas es el PAU donde la superficie de menor nivel de sellado es de más del $70 \%$, con un sellado de parcela de clase 0 . Inicialmente, y a la espera de un análisis de la evolución posterior al año de la fuente, puede apreciarse que obedece en gran medida a la existencia en dicho PAU del Parque urbano Felipe VI, que viene a ser algo más de la mitad de toda la superficie planimetrada del PAU (Figura 5). La conformación viaria que cierra Valdebebas, contiene amplios espacios verdes de protección de vías, en consonancia con la jerarquía de las mismas: M40-R2M12 y M11. Por otro lado, la existencias de numerosas manzanas, planificadas como residenciales, pero vacantes o en fase de obras, arroja también un bajo índice de sellado, que tan solo será coyuntural, pues hasta que no finalicen las obras de fábrica, no se conocerá la cubierta definitiva. Todo ello, unido a la ubicación de las instalaciones de la Ciudad Deportiva del Club Real Madrid justifica los bajos valores, que este PAU ofrece frente al resto de los desarrollos urbanos analizados.

\section{Figura 5. Fotografía de Valdebebas: modelo urbano con amplios espacios abiertos}

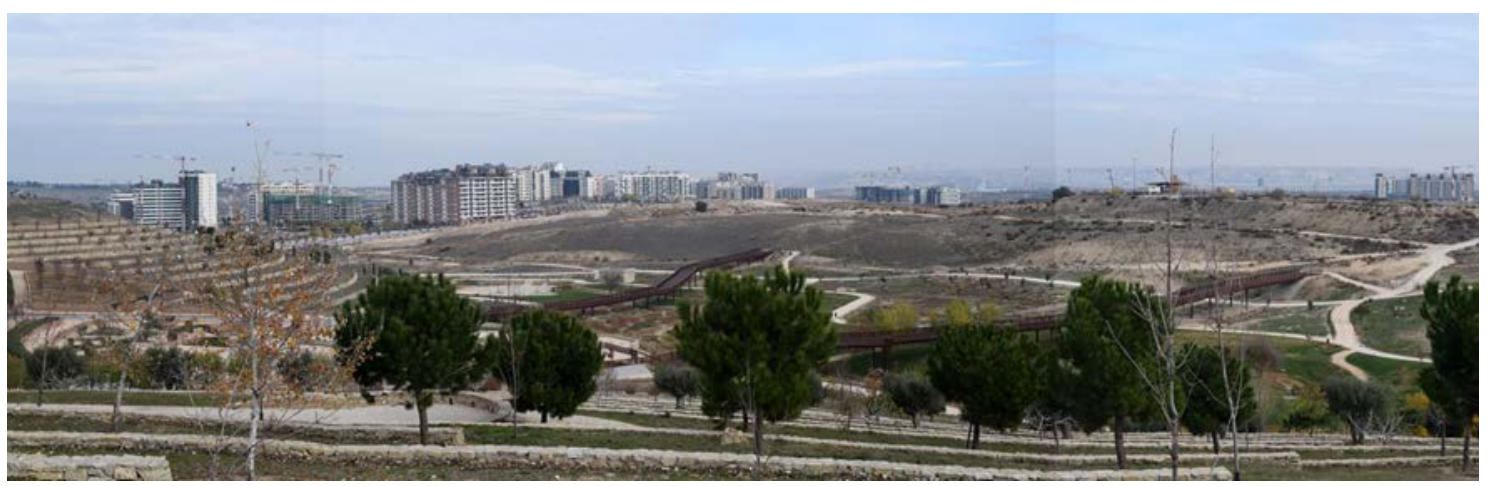

Fuente: Pérez González, 10 de diciembre de 2015 
3. La relación entre el nivel de sellado y la geometría parcelaria de los diferentes PAUS muestra que tres de los cuatro espacios urbanos presentan un tamaño medio de parcela muy similar (1,84-1,24 ha) (Tabla 2), con la excepción de Valdebebas, que alcanza un 4,5 ha. Esta peculiaridad está en consonancia con la menor densidad de ocupación de la función meramente residencial.

4. La superficie sellada difiere sensiblemente según la escala de trabajo utilizada, la fuente y la metodología utilizada. No obstante, en ningún caso la discordancia supera el $10 \%$ y, en dos PAUS, esta diferencia no alcanza el $5 \%$.

5. Queda generada la base de datos necesaria para la adición de indicadores, para su posterior explotación e interpretación de la evaluación del sellado, de su naturaleza y consecuencias. Es importante señalar, que, desde una perspectiva evolutiva de la dinámica urbana, hemos fijado un comienzo para futuras comparaciones, del mismo lugar, y también una referencia para contrastar con otros espacios urbanos, de similar dinámica.

En la tercera fase del trabajo, y una vez establecido los porcentajes de sellado, se seleccionaron ocho muestras de suelos representativas de los distintos PAUS, con el fin de analizar qué tipo de suelos están desapareciendo por sellado. Las ocho muestras analizadas corresponden a Luvisoles háplicos/cálcicos, ya que es la asociación que alcanza más extensión en el norte y este del municipio de Madrid (Tabla 3).

Tabla 3. Análisis de los suelos

\begin{tabular}{|c|c|c|c|c|c|c|c|c|c|c|c|}
\hline Muestra & $\begin{array}{c}\text { Grava } \\
\%\end{array}$ & $\begin{array}{c}\text { Ar.gr } \\
\%\end{array}$ & $\begin{array}{c}\text { Ar.fin } \\
\%\end{array}$ & $\underset{\%}{\operatorname{Limo}}$ & $\begin{array}{c}\text { Arcill } \\
\%\end{array}$ & $\begin{array}{c}\mathrm{pH} \\
\mathrm{H} 2 \mathrm{O}\end{array}$ & $\begin{array}{l}\mathrm{pH} \\
\mathrm{KCl}\end{array}$ & $\begin{array}{c}\text { Carbon } \\
\text { atos\% }\end{array}$ & $\begin{array}{c}\text { MO } \\
\%\end{array}$ & C.E & $\begin{array}{c}\text { Arena } \\
\text { total }\end{array}$ \\
\hline 1 Montecarmelo & 14.68 & 28.90 & 33.97 & 30.10 & 7.03 & 7.4 & 7.0 & 16.80 & 8.29 & 0.9 & 62.87 \\
\hline 2 Monecarmelo & 27.12 & 29.00 & 33.20 & 29.38 & 8.42 & 7.7 & 7.2 & 23.53 & 1.93 & 0.85 & 62.20 \\
\hline 3 Montecarmelo & 22.48 & 36.80 & 42.85 & 5.08 & 15.27 & 6.9 & 5.5 & 1.03 & 0.67 & 0.21 & 79.65 \\
\hline 4 Las Tablas & 26.96 & 46.10 & 36.02 & 4.93 & 12.95 & 6.8 & 5.5 & 1.00 & 0.02 & 0.42 & 82.12 \\
\hline 5 Las Tablas & 15.46 & 33.80 & 51.15 & 5.05 & 10.00 & 7.6 & 6.8 & 1.10 & 1.13 & 0.26 & 84.95 \\
\hline 6 Sanchinarro & 11.72 & 44.80 & 30.35 & 6.20 & 18.65 & 7.1 & 5.8 & 0.92 & 1.13 & 0.21 & 75.15 \\
\hline 7 Sanchinarro & 15.66 & 47.90 & 28.67 & 7.25 & 16.18 & 7.6 & 6.9 & 1.22 & 1.82 & 0.29 & 76.57 \\
\hline 8 Valdebebas & 38.16 & 53.20 & 20.75 & 6.03 & 20.02 & 6.8 & 5.7 & 0.92 & 3.09 & 0.28 & 73.95 \\
\hline
\end{tabular}

Fuente: elaboración propia 
Son suelos de textura franco arenosa o arenosa franca, con un porcentaje elevado en arenas gruesas, lo que implica que tienen una alta permeabilidad. Solo la muestra de Valdebebas tiene una textura más fina, franco arcillo arenosa, por lo que la permeabilidad es más baja. Los contenidos en materia orgánica no son muy altos, salvo en los suelos que mantienen los pinares (muestra 1, Tabla 3). Los pH están ligados al contenido en carbonatos, de forma que aquellos perfiles ricos en carbonato cálcico tienen $\mathrm{pH}$ alcalinos y, el resto, tienen un $\mathrm{pH}$ neutro o ligeramente ácido.

Su perdida por sellado implica un incremento en el riesgo de escorrentía dada la alta permeabilidad de los suelos arenosos. Además, el ciclo biogeoquímico queda interrumpido con la considerable pérdida de nutrientes y la disminución como sumidero de carbono.

\section{Conclusiones}

El sellado de suelo se analiza en Madrid a diversas escalas, desde 1:50 000 a 1:10 000, utilizando una amplia base de datos espaciales, como son imágenes del satélite Spot-5, fotografías aéreas, cartografía temática y parcelario urbano, combinando técnicas de teledetección y SIG.

El análisis de menor escala realizado con el satélite Spot-5 resulta adecuado para hacer un seguimiento del sellado del suelo. Aun cuando su resolución espacial sea menor que la obtenida mediante fotografías aéreas, la facilidad de actualización casi continua, su accesibilidad y gratuidad para los organismos públicos lo hace idóneo para este tipo de estudios. En todo caso es fundamental unir ambas fuentes.

La comparación de estos datos con los extraídos del parcelario y fotografías aéreas permite el análisis multiescalar desde lo más general, a 1:50 000, al mayor detalle del 1:30 000 y 1:10 000 .

Aunque los resultados obtenidos sobre el sellado del suelo con diferentes fuentes de información y escalas dispares no ofrezca en todos los casos la similitud deseada, las diferencias no alcanzan el $10 \%$ de la superficie, valores que se reducen considerablemente al 1-5\% en los espacios urbanos más compactados y, por consiguiente, con mayor pérdida de suelo.

Los recientes proyectos urbanísticos del norte de Madrid suponen un sellado de suelos que oscila entre un $60 \%$ (Montecarmelo) y un $36 \%$ (Valdebebas). Los valores obtenidos mediante imágenes de satélite son levemente superiores a los calculados a partir del análisis parcelario, pues éste permite una mayor discriminación espacial entre categorías.

La pérdida por sellado en el norte y noreste de Madrid afecta a las propiedades del suelo, especialmente a la escorrentía debido a la textura arenosa de los suelos y, a la larga, a la absorción de $\mathrm{CO}^{2}$ y a la diversidad biológica. 
Aun cuando se urbanice, si se dejan amplios espacios verdes como está sucediendo en muchos PAUS, se mitigan los efectos adversos de sellado y, si se plantan especies vegetales del ámbito mediterráneo se disminuye la contaminación del aire, problema grave en la ciudad de Madrid. Por eso es importante al realizar la cartografía subdividir el sellado teniendo en cuenta su densidad, como ya han señalado otros autores (Pascual et al, 2005; Gardi et al, 2011; Valera et al., 2011 y Pulido et al., 2014).

Puede concluirse, sin ánimo de cierre de polémica, que los nuevos desarrollos urbanos del norte de Madrid incorporan espacios "libres" de sellado, que aportan valor añadido al suelo construido, tanto desde el punto de vista económico como medioambiental. 


\section{Bibliografía}

Al-Dousari, A. M., Misak, R., y Shahid, S. (2000). Soil compaction and sealing in Al-Salmi area, Western Kuwait. Land Degradation and Development, 11(5), 401-418.

Artmann, M. (2014). Assessment of soil sealing management responses, strategies, and targets toward ecologically sustainable urban land use management. Ambio, 43(4), 530-541.

Assouline, S., y Mualem, Y. (2006). Runoff from heterogeneous small bare catchments during soil surface sealing. Water Resources Research, 42(12).

Brandis, D. (2014). La producción inmobiliaria de la periferia madrileña (1991-2013). En J. J. Michelini (Ed.), Desafíos metropolitanos (pp. 169-189). Madrid: Catarata.

Burghardt, W. (2006). Soil sealing and soil properties related to sealing. Geological Society of London, Special Publications, 266, 117-124.

Chen, L., Sela, S., Svoray, T., y Assouline, S. (2013). The role of soil-surface sealing, microtopography, and vegetation patches in rainfall-runoff processes in semiarid areas. Water Resources Research, 49(9), 5585-5599.

Eilers, L. (1973). Soil sealing method. US Patent 3,772,893.

European Commission (2012). Guidelines on best practice to limit, mitigate or compensate soil sealing (Working Document). Brussels.

FAO (1979). A provisional methodology for soil degradation assessment. Roma: FAO.

García Alvarado, J. Mª Pérez González, Mª E., y García Rodríguez, Ma P. (2014). Revisión del concepto de sellado de suelos y propuesta de tipología urbana. Anales de Geografía de la Universidad Complutense, 34, 87-103.

García Rodríguez, P, y Pérez González, M. E. (2007). Changes in soil sealing in Guadalajara (Spain): Cartography with Landsat images. Science of the Total Environment, 378(1-2), 209-213.

García Rodríguez, Mª P., y Pérez González, Mª E. (2011). Sellado de fluvisoles en la Comunidad de Madrid análisis a partir de imágenes Landsat. Anales de Geografía de la Universidad Complutense, 31, 125-137.

García Rodríguez, Ma P., y Pérez González, Ma E. (2014). Análisis multitemporal del urbanismo expansivo en el Corredor del Henares. Aportación de las imágenes de satélite. Estudios Geográficos, LXXV(277), 597-618.

García, P., Pérez, M. E., y Guerra, A. (2014). Using TM images to detect soil sealing change in Madrid (Spain). Geoderma, 214-215, 135-140. 
García, P., y Pérez M. E. (2016). Mapping of soil sealing by vegetation indexes and built-up index: A case study in Madrid (Spain). Geoderma, 268, 100-107.

Gardi, C., Montanarella, L., Tóth, G., Palmieri, A., Martino, L., y Erhard, M. (2011). The Assessment of Soil Sealing and Land Take in Europe. En G. Tóth y T. Németh (Eds.), Land Quality and Land Use Information in the European Union (pp. 173-186). European Comission, Publications Office of the European Union.

Govers, G., y Poesen, J. (1986). "A field-scale study of surface sealing and compaction on loam and sandy loams soils. Part I. Spatial variability of soil surface sealing and crusting. Assessment of Soil Surface Sealing and Crusting. En F. Callebaut, D. Grabiéls y M. Broodt (Eds.), A field-scale study of surface sealing (pp. 171-182). Actas del Congreso celebrado en Ghent (Bélgica).

Heil, J. W., Juo, A. S. R., y Mcinnes, K. J. (1997). Soil properties influencing surface sealing of some sandy soils in the Sahel. Soil Science, 162(7), 459-469.

Jakab, G., Németh, T., Csepinszky, B., Madarász, B., Szalai, Z., y Kertész, Á. (2013). The influence of short term soil sealing and crusting on hydrology and erosion at balaton uplands, Hungary. Carpathian Journal of Earth and Environmental Sciences, 8(1), 147-155.

Ministerio de Fomento. Instituto Geográfico Nacional. Disponible en http://www.ign.es/web/ign/portal

Mualem, Y., y Assouline, S. (1989). Modeling soil seal as a nonuniform layer. Water Resources Research, 25(10), 2101-2108.

Mualem, Y., Assouline, S., y Rohdenburg, H. (1990). Rainfall induced soil seal (A) A critical review of observations and models. Catena, 17(2), 185-203.

Munafo, M., Norero, C., Sabbi, A., y Salvati, L. (2010). Soil Sealing in the Growing City: A Survey in Rome, Italy. Scottish Geographical Journal, 126(3), 153-161.

Munafo, M., Salvati, L., y Zitti, M. (2013). Estimating soil sealing rate at national level. Italy as a case study. Ecological Indicators, 26, 137-140.

Netzband, M. y Meinel, G. (1998). Identifying urban soil sealing by high resolution remote sensing methods. En J. Breuste, H. Feldmand y O. Uhlman (Eds.), Urban Ecology (pp. 451-455). SpringerVerlag.

Pascual, J. A., Año, C., Sanjaime, V., y Sánchez, J. (2005). Estimating soil sealing rates in Mediterranean coastal environments. Preliminary results for Castellon, Spain. Advances in Geoecology, 36, 339-346.

Poesen, J., y Govers, G. (1986). A field-scale study of surface sealing and compaction on loam and sandy loam soils. Part II. Impact of soil surface sealing and compaction on water erosion processes. 
Assessment of Soil Surface Sealing and Crusting. En F. Callebaut, D. Grabiéls y M. Broodt (Eds.), A field-scale study of surface sealing (pp. 171-182). Actas del Congreso celebrado en Ghent (Bélgica).

Prokop, G., Jobstmann, H., y Schönbauer, A. (2011). Overview of best practices for limiting soil sealing or mitigating its effects in EU-27. Brussels: European Communities.

Pulido Moncada, M., Gabriels, D., Lobo, D., De Beuf, K., Figueroa, R., y Cornelis, W. M. (2014). A comparison of methods to assess susceptibility to soil sealing. Geoderma, 226-227(1), 397-404.

Ramos, M. C., Nacci, S., y Pla, I. (2000). Soil sealing and its influence on erosion rates for some soils in the Mediterranean area. Soil Science, 165(5), 398-403.

Römkens, M. J. M., Prasad, S. N., y Gerits, J. J. P. (1997). Soil erosion modes of sealing soils: A phenomenological study. Soil Technology, 11(1), 31-41.

Scalenghe, R., y Marsan, F. A. (2009). The anthropogenic sealing of soils in urban areas. Landscape and Urban Planning, 90(1-2), 1-10.

Singer, M. J., y Le Bissonnais, Y. (1998). Importance of surface sealing in the erosion of some soils from a Mediterranean climate. Geomorphology, 24(1), 79-85.

Ungaro, F., Calzolari, C., Pistocchi, A., y Malucelli, F. (2014). Modelling the impact of increasing soil sealing on runoff coefficients at regional scale: a hydropedological approach. Journal of Hydrology and Hydromechanics, 62(1), 33-42.

University of Maryland. Global Land Cover Facility. Disponible en http://glcf.umd.edu/data/landsat/

U.S. Geological Service. GloVis. Disponible en http://glovis.usgs.gov/

Valentin, C. (1994). Sealing, crusting and hardsetting soils in sahelian agriculture. En H. B. So, G.

D. Smith, S. R. Raine, B. M. Schafer y R. J. Loch (Eds.), Second International Symposium on Sealing, Crusting and Hardsetting Soils: Productivity and Conservation (pp. 53-76). Australian Society of Soil Science Inc.

Valera, A., Añó, C., y Sánchez, J. (2011). Cincuenta años (1956-2006) de crecimiento urbano y degradación de suelos por sellado antropogénico en el término municipal de Valencia. Anales de Geografía de la Universidad Complutense, 31(2), 177-191.

Xiao, R., Su, S., Zhang, Z., Qi, J., Jiang, D., y Wu, J. (2013). Dynamics of soil sealing and soil landscape patterns under rapid urbanization. Catena, 109, 1-12.

Zhao, D., Li, F., Wang, R., Yang, Q., y Ni, H. (2012). Effect of soil sealing on the microbial biomass, $\mathrm{N}$ transformation and related enzyme activities at various depths of soils in urban area of Beijing, China. Journal of Soils and Sediments, 12(4), 519-530. 